\title{
Multi-soliton Solutions for a Class of Fifth-order Evolution Equations
}

\author{
Rafat Ashorman \\ Computer Science Department \\ Zarqa University, Jordan \\ Rafat_sh@zu.edu.jo
}

\begin{abstract}
By employing a simplified bilinear method, a class of generalized fifth-order KdV(gfKdV) equations which arise in nonlinear lattice, plasma physics and ocean dynamics are investigated. With the aid of symbolic computation, both solitary wave soluhons and multiplesoliton solutions are obtained. These new exact solutions wilt extend previous results and help us explain the properties of nonlinear solitary waves in many physical models in shallow water. Parametric analysis is carried out in order to illustrate that the soliton amplitude, width and velocity are affected by the coefficient parameters in the equation.
\end{abstract}

Keywords: Multiple soliton solutions; Fifth-order evolution equations; Cole-Hopf transformation; Hirota bilinear method

\section{Introduction}

In this paper, the simplified version of Hirota's nethod is applied to construct multi-soliton solutions of a class of fifth-order evolution equations of the form

$u_{t}+\alpha u u_{3 x}+\beta u_{x} u_{2 x}+\gamma u^{2} u_{x}+u_{5 x}$

where the coefficients $\alpha, \beta, \gamma$ are real-valued parameters that are physically meaningful.

This class inclades the Lax [9], Sawada-Kotera (SK) [10, 13, 14], Kaup-Kupershmidt (KK) [11] and Ito equations 17$]$ As the constants $\alpha, \beta, \gamma$ take different values, the properties of Eq. (1) drastically change Eor instance, the Lax equation with $\alpha=10, \beta=20$ and $\gamma=30$, and the SK equation where $\gamma=\beta=\gamma=5$, are completely integrable. These two equations have $\mathrm{N}$-soliton solutions and an infinite set of conservation laws. The KK equation, with $\alpha=$ $10, \beta=25=10$ and $\gamma=20$, is also known to be integrable [8] and to have bilinear representations. A fourth equation in the class (1) is the Ito equation with $\alpha=3, \beta=6$ and $\gamma=2$, which is not completely integrable, but has a limited number of conservation laws [7].

Obyiously, for arbitrary values of with $\alpha, \beta$ and $\gamma$ Eq. (1) is not completely integrable, and therefore does not admit solitons. This does, however, not exclude the existence of closedform solitary-wave solutions. Also, with scales on $\mathrm{u}, \mathrm{x}$ and $\mathrm{t}$, the named equations cannot be transformed into one another; they are fundamentally different.

To our knowledge, there have been no discussions on Eq. (1) in the present of the coefficient parameters $\alpha, \beta, \gamma$. All the existing works deal with special cases of Eq. (1). Considering such insufficiency, we will apply a simplified bilinear method to investigate the $\mathrm{N}$-soliton solutions for Eq. (1). The results obtained for the multiple regular soliton solutions 
agree well with the results in the previous works and this the first work for the multiple singular soliton solutions.

In this work, the Cole-Hopf transformation method combined with Hirota's bilinear method is used to study the equations in class (1). Our goal from applying this method is to construct multiple regular soliton solutions and multiple singular soliton solutions. The introduced simplified algorithm derives the auxiliary functions, obtained in Hirota's method, without using the bilinear forms.

\section{Soliton Solutions for Class (1)}

\subsection{Cole-Hopf Transformation Method}

To derive $\mathrm{N}$-soliton solutions of any completely integrable equation, we will mainly ase the Hirota's direct method. The Hirota method $[4,12,5,11]$ relies on a transformation for considered equation to a bilinear form. The bilinear forms are usually used to enable us deriving the auxiliary function. It is remarkable to mention that it is not easy for us to find the bilinear form for many equations and sometimes it requires the introduction of new dependent and sometimes even independent variables. However, Hereman et at. [15, 16, 3], formally introduced the simpli-fied algorithm to derive the auxiliary functions without using the bilinear forms. The Cole-Hopf transformation method combined with the simplified Hirota's sense is a powerful method to deter-mine mulaple soliton solutions and multiple singular soliton solutions for integrable systems. We summarize the necessary steps of the method [1, $2,6,19,18,17]$ :

We first substitute

$\mathrm{u}(\mathrm{x}, \mathrm{t})=\mathrm{e}^{\mathrm{k}_{\mathrm{i}} \mathrm{x}-\mathrm{w}_{\mathrm{i}} \mathrm{t}}$

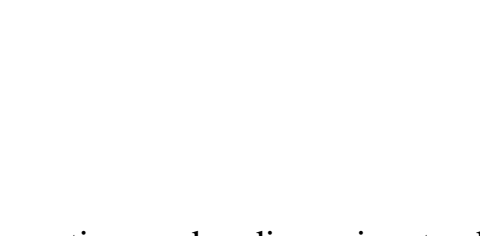

into the linear terms of the equation under discussion to determine the dispersion relation between $\mathrm{k}_{\mathrm{i}}, \mathrm{r}_{\mathrm{i}}$ and $\mathrm{w}_{\mathrm{i}}$. To-obtam the singlesoliton solution, we use a suitable transformation method, such as the Cole-Hopf transformation

$\mathrm{u}(\mathrm{x}, \mathrm{t})=\mathrm{R}\left(\ln \mathrm{l}_{\mathrm{x} x}\right.$

into the equation under discussion, where the auxiliary function $f(x, t)$ is given by

$f(x, t)=1+e^{\theta_{1}}$

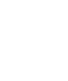

Where

$\theta_{\mathrm{i}}=\mathrm{k}_{\mathrm{i}} \mathrm{x} \rightarrow \mathrm{w} \mathrm{i}, \mathrm{i}=1,2, \ldots, \mathrm{N}$

Then solving the resulting equation to determine the numerical value for $\mathrm{R}$. The $\mathrm{N}$-soliton solutions can be obtained by using the following forms for $f(x, t)$ :

For dispersion relation, we use

$u(x, t)=e^{k_{i} x-w_{i} t}$

For single soliton solution, we use 
$f(x, t)=1+e^{\theta_{1}}$

- For two-soliton solutions, we use

$f(x, t)=1+e^{\theta_{1}}+e^{\theta_{2}}+a_{12} e^{\theta_{1}+\theta_{2}}$

- For three-soliton solutions, we use

$f(x, t)=1+e^{\theta_{1}}+e^{\theta_{2}}+e^{\theta_{3}}+a_{12} e^{\theta_{1}+\theta_{2}}+$

$\mathrm{a}_{13} \mathrm{e}^{\theta_{1}+\theta_{3}}+\mathrm{a}_{23} \mathrm{e}^{\theta_{2}+\theta_{3}}+\mathrm{a}_{123} \mathrm{e}^{\theta_{1}+\theta_{2}+\theta_{3}}$

Notice that if we find that $\mathrm{a}_{123}=\mathrm{a}_{12} \mathrm{a}_{13} \mathrm{a}_{23}$ then three-soliton solutions are obtained. The determination of three-soliton solutions confirms the fact that $\mathrm{N}$-soliton solutions exist for any order.

However, for the multiple singular soliton solutions, we follow the following steps:

- For dispersion relation, we use

$\mathrm{u}(\mathrm{x}, \mathrm{t})=\mathrm{e}^{\mathrm{k}_{\mathrm{i}} \mathrm{x}-\mathrm{w}_{\mathrm{i}} \mathrm{t}}$

- For single soliton, we use

$f(x, t)=1-e^{\theta_{1}}$

- For two-solution solutions we use

$f(x, t)=1-e^{\theta_{1}}-e^{\theta_{2}}+a_{12} e^{\theta_{1}+\theta_{2}}$

- For three-soliton solutions we use

$\mathrm{f}(\mathrm{x}, \mathrm{t})=1-\mathrm{e}^{\theta_{1}}-\mathrm{e}^{\theta_{2}}-\mathrm{e}^{\theta_{3}}+\mathrm{a}_{2} \mathrm{e}^{\theta_{1}+\theta_{2}}+$

$\mathrm{a}_{13} \mathrm{e}^{\theta_{1}+\theta_{3}}+\mathrm{a}_{23} \mathrm{e}^{\theta_{2}+\theta_{3}}-\mathrm{a}_{123} \mathrm{e}^{\theta_{1}+\theta_{2}+\theta_{3}}$

\subsection{Multiple Soliton Solutions}

We first substitute

$\mathrm{u}(\mathrm{x}, \mathrm{t})=\mathrm{e}^{\mathrm{k}_{\mathrm{i}} \mathrm{x}-\mathrm{w}_{\mathrm{i}} \mathrm{t}}$

into the Trmear terms of Eq.(1) to determine the dispersion relation as follows
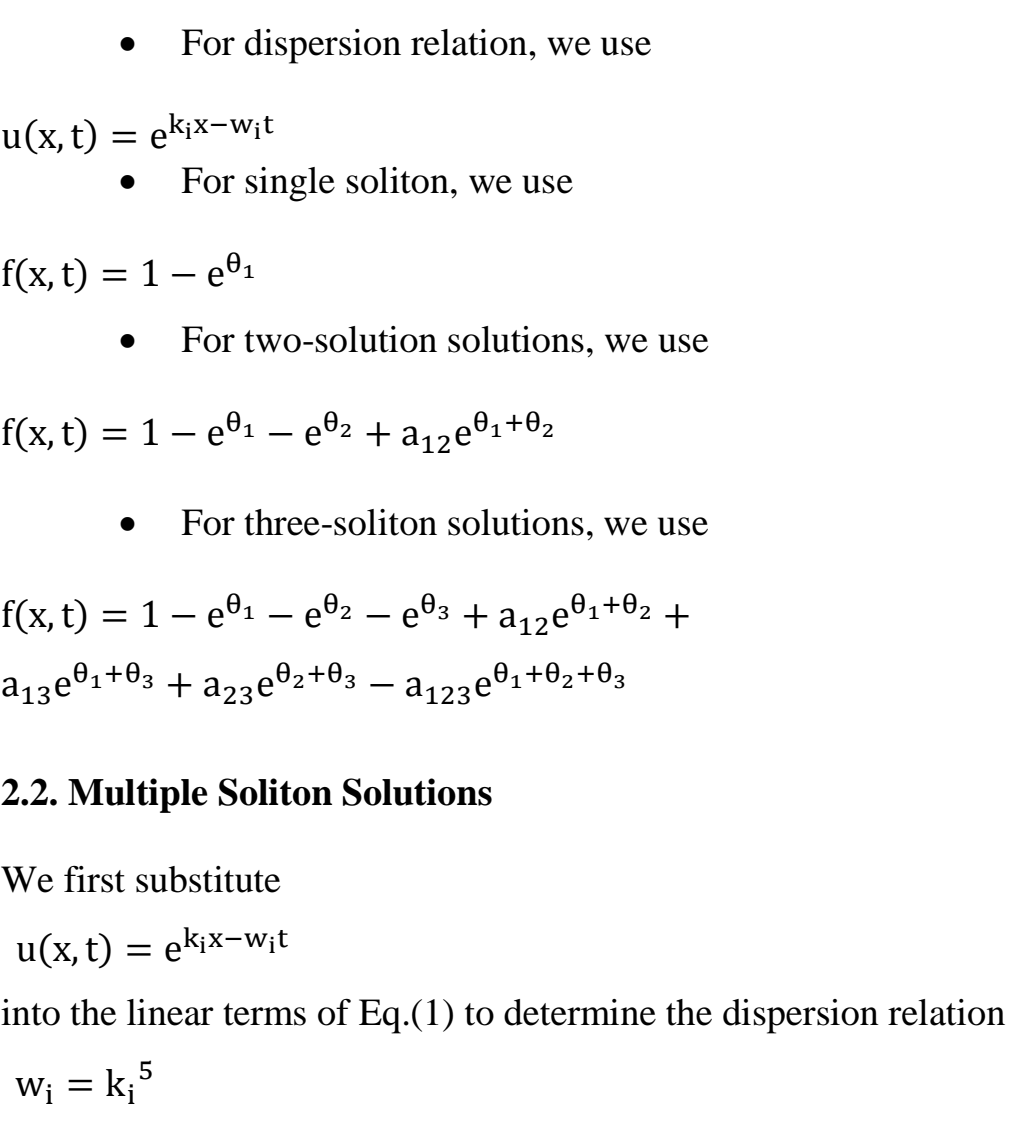

2

It is obvious that the dispersion relation (3) depends on the coefficient of $\mathrm{x}$. As a result we obtain

$$
\theta_{\mathrm{i}}=\mathrm{k}_{\mathrm{i}} \mathrm{x}-\mathrm{k}_{\mathrm{i}}^{5} \mathrm{t} \text {. }
$$

The single soliton solution of Eq. (1) is assumed to be

$$
\mathrm{u}(\mathrm{x}, \mathrm{t})=\mathrm{R}(\ln f)_{\mathrm{xx}}
$$


where the auxiliary function $\mathrm{f}(\mathrm{x} ; \mathrm{t})$ is given by

$$
\mathrm{f}(\mathrm{x}, \mathrm{t})=1+\mathrm{e}^{\theta_{1}}=1+\mathrm{e}^{\mathrm{k}_{1} \mathrm{x}-\mathrm{k}^{5}{ }_{1}^{\mathrm{t}}}
$$

Substitute (5) into Eq. (1) and solve the system for R. We find the solvability condition

$$
\alpha^{2}+\beta \alpha-10 \gamma=0
$$

is necessary to obtain

$$
\mathrm{R}=\frac{60}{\alpha+\beta}
$$

By substituting $f(x, t)$ into (5), we obtain the single soliton solution

$\mathrm{u}(\mathrm{x}, \mathrm{t})=\frac{60}{\alpha+\beta} \mathrm{k}_{1}^{2} \frac{\mathrm{e}^{\theta_{1}}}{\left(1+\mathrm{e}^{\theta_{1}}\right)^{2}}$

$=\frac{60}{\alpha+\beta} \mathrm{k}_{1}{ }^{2} \frac{\mathrm{e}^{\mathrm{k}_{1} \mathrm{x}-\mathrm{k}^{5}{ }_{1} \mathrm{t}}}{\left(1+\mathrm{e}^{\mathrm{k}_{1} \mathrm{x}-\mathrm{k}^{5}{ }_{1} \mathrm{t}}\right)^{2}}$

The solution in (9) can be written as

$$
\mathrm{u}(\mathrm{x}, \mathrm{t})=\frac{15}{\alpha+\beta} \mathrm{k}_{1}{ }^{2} \operatorname{sech}^{2}\left(\frac{\theta_{1}}{2}\right)
$$

which is a bell-shaped solitary wave solution for Eq. (1) For two-soliton solutions, we set

$$
\mathrm{f}(\mathrm{x}, \mathrm{t})=1+\mathrm{e}^{\theta_{1}}+\mathrm{e}^{\theta_{2}}+\mathrm{a}_{12} \mathrm{e}^{\theta_{1}+\theta_{2}}
$$

where $\theta_{1}$ and $\theta_{2}$ are defined in (4). Using (11) in

$\mathrm{u}(\mathrm{x}, \mathrm{t})=\frac{60}{\alpha+\beta}(\ln \mathrm{f})_{\mathrm{xx}}$

and substituting the result in Eq.(1), we obtain the phase shift by

$\mathrm{a}_{12}=\mathrm{K}\left(\alpha, \beta, \mathrm{k}_{1} \mathrm{k}_{2}\right)$

The three-soliton solutions can be obtained by using

$f(x, t)=1+e^{\theta_{1}}+e^{\theta_{2}}+e^{\theta_{3}}+a_{12} e^{\theta_{1}+\theta_{2}}+$ $\mathrm{a}_{13} \mathrm{e}^{\theta_{1}+\theta_{3}}+\mathrm{a}_{23} \mathrm{e}^{\theta_{2}+\theta_{3}}+\mathrm{a}_{123} \mathrm{e}^{\theta_{1}+\theta_{2}+\theta_{3}}$

where $\mathrm{a}_{\mathrm{ij}}$ S given by (13). Substituting (14) and (12) into Eq.(1), we find always that

$a_{123}=a_{12} a_{13} a_{23}$

Since $(15)$ holds, we can use the results indicated in $(6,7,8,9)$ to conclude that the $\mathrm{N}$-soliton solutions for Eq.(1) can be obtained for finite $\mathrm{N}$, where $\mathrm{N} \geq 1$.

\subsection{Multiple Singular Soliton Solutions}

To obtain a single singular soliton solution, we substitute $u(x, t)=e^{k_{i} x-w_{i} t}$ 
into the linear terms of Eq.(1). This gives the dispersion relation by

$\mathrm{w}_{\mathrm{i}}=\mathrm{k}_{\mathrm{i}}{ }^{5}$

and as a result we obtain

$\theta_{\mathrm{i}}=\mathrm{k}_{\mathrm{i}} \mathrm{x}-\mathrm{k}_{\mathrm{i}}^{5} \mathrm{t}$.

The singular single soliton solution of Eq (1) is assumed to be

$\mathrm{u}(\mathrm{x}, \mathrm{t})=\mathrm{R}(\ln f)_{\mathrm{xx}}$

where the auxiliary function $f(x, t)$ is given by

$f(x, t)=1+e^{\theta_{1}}=1+e^{k_{i} x-k^{5} t}$

Substituting (19)-(20) into Eq.(1) and solving for $\mathrm{R}$ we find $\mathrm{R}=60 /(\alpha+\beta)^{\text {Now, }}$, Eq.(19) gives the single singular soliton solution

$\mathrm{u}(\mathrm{x}, \mathrm{t})=-\frac{60}{\alpha+\beta} \mathrm{k}_{1}{ }^{2} \frac{\mathrm{e}^{\theta_{1}}}{\mathrm{e}^{\theta_{1-1}}}$,

and so

$\mathrm{u}(\mathrm{x}, \mathrm{t})=\frac{15}{\alpha+\beta} \mathrm{k}_{1}^{2} \operatorname{csch}^{2}\left(\frac{\theta_{1}}{2}\right)$

where $\theta_{1}$ is defined by (18).

Multiple singular soliton solutions for Eq (1) car be expressed in the following form:

$u(x, t)=\frac{60}{\alpha+\beta}(\ln f)_{x x}$.

To determine the two singolar soliton solutions explicitly, we substitute

$f(x, t)=1-e^{\theta_{1}}-e^{\theta_{2}}+a_{12} e^{\theta_{1}+\theta_{2}}$

where $\theta_{1}$ and $\theta_{2}$ are defined in (18), into (21). As a result we get

$\mathrm{a}_{\mathrm{ij}}=\mathrm{K}\left(\alpha, \beta, \mathrm{k}_{1}, \mathrm{k}_{2}\right)$.

For the singular three-soliton solutions we use

$\mathrm{f}(\mathrm{x}, \mathrm{t})=1-\mathrm{e}^{\theta_{1}}-\mathrm{e}^{\theta_{2}}-\mathrm{e}^{\theta_{3}}+\mathrm{a}_{12} \mathrm{e}^{\theta_{1}+\theta_{2}}+$

$\mathrm{a}_{13} \mathrm{e}^{\theta_{1}+\theta_{3}}+\mathrm{a}_{23} \mathrm{e}^{\theta_{2}+\theta_{3}}-\mathrm{a}_{123} \mathrm{e}^{\theta_{1}+\theta_{2}+\theta_{3}}$,

where $\theta_{i}(j=1,2,3)$ are defined in $(18)$ and $a_{i j}$ are defined in (15). Proceeding as before, we obtain always

$\mathrm{a}_{123}=\mathrm{a}_{12} \mathrm{a}_{13} \mathrm{a}_{23}$

The singular three-soliton solution can be obtained explicitly using (21) for the function $\mathrm{f}$ in (23).

\subsection{Analysis of the Parameters}

The solution (10) gives a profile of bell-shaped solitary wave with soliton amplitude amp and width $\Delta$ can be expressed as 
$\operatorname{amp}=\frac{15}{|\alpha+\beta|} \mathrm{k}_{1}{ }^{2}, \quad \Delta=\frac{2}{\left|\mathrm{k}_{1}\right|}$

With the characteristic-line method $[2,6]$, the characteristic line for each solitary wave can be defined by

$\mathrm{x}-\mathrm{k}_{\mathrm{i}}^{4}=0, \quad \mathrm{i}=1,2,3, \ldots$,

which can be derived from relations (3) and (4). Correspondingly, the velocity $\mathrm{v}$ of each solitary wave can be expressed by

$$
\mathrm{v}_{\mathrm{i}}=\mathrm{k}_{\mathrm{i}}^{4}
$$

Further, the absolute value of velocity $\mathrm{v}$ determines the speed, namely, velocity, in magnitude, and propagation direction of soliton is decided by the sign of $\mathrm{v}$.

We now analytically examine the effects of the parameters $\alpha, \beta, \gamma$ on the behavior of the solitary waves. The soliton amplitude amp is dependent on the ratio of 15 to $|d+\beta|$. The solitonic amplitude increases with an increase in the ratio $15 \gamma|\alpha+\beta|$. Howeyer, Expression (26) indicates that the propagation velocity of the solitary wave is not influenced by the coefficient parameters $\alpha, \beta$ and $\gamma$.

\section{Applications}

In this section we will present some exanples and testcases.

\subsection{The Sawada-Kotera Equation}

Consider the Sawada-Kotera equation

$u_{t}+15 u u_{3 x}+15 u_{x} u_{2 x}+45 u^{2} u_{x}+u_{5 x}=0$

We can verify that the parameters $\alpha, \beta$ and $\gamma$ satisfy the solvability Condition (7). So, $\mathrm{R}=2$ and the one-soliton solution is given by

$\mathrm{u}(\mathrm{x}, \mathrm{t})=\frac{1}{2} \mathrm{k}_{1}{ }^{2} \operatorname{sech}^{2}\left(\frac{\theta_{1}}{2}\right)$

and the singular one-s6liton solution is

$\mathrm{u}(\mathrm{x}, \mathrm{t})=\frac{1}{2} \mathrm{k}_{1}{ }^{2} \operatorname{csch} \mathrm{sen}^{2}\left(\frac{\theta_{1}}{2}\right)$,

where $\theta_{1}$ is defined in (4).

Using (11) in

$$
\mathrm{u}(\mathrm{x}, \mathrm{t})=2(\ln \mathrm{f})_{\mathrm{xx}}
$$

and substituting the result in Eq. (27), we obtain the phase shift by

$$
\mathrm{a}_{12}=\frac{\left(\mathrm{k}_{1}{ }^{2}-\mathrm{k}_{1} \mathrm{k}_{2}+\mathrm{k}^{2}{ }_{2}\right)\left(\mathrm{k}_{1-} \mathrm{k}_{2}\right)^{2}}{\left(\mathrm{k}_{1}{ }^{2}+\mathrm{k}_{1} \mathrm{k}_{2}+\mathrm{k}^{2}{ }_{2}\right)\left(\mathrm{k}_{1+} \mathrm{k}_{2}\right)^{2}}
$$

We can generalize for other phase shifts by 
$a_{i j}=\frac{\left(k_{i}^{2}-k_{i} k_{j}+k_{j}^{2}\right)\left(k_{i-} k_{j}\right)^{2}}{\left(k_{i}^{2}+k_{i} k_{j}+k^{2}{ }_{2}\right)\left(k_{i+} k_{j}\right)^{2}}, \quad 1 \leq i \leq j \leq 3$.

Using (11) and (14) in (28), we can obtain the closed-form two-soliton and three-soliton solutions.

\subsection{The Lax Equation}

The Lax equation is given by

$$
+10 u u_{3 x}+20 u_{x} u_{2 x}+30 u^{2} u_{x}+u_{5 x}=0
$$

The parameters $\alpha, \beta$ and $\gamma$ satisfy the solvability Condition (7), and as a result, $R=2$. For the Lax equation, the one-soliton solution is given by

$\mathrm{u}(\mathrm{x}, \mathrm{t})=\frac{1}{2} \mathrm{k}_{1}^{2} \operatorname{sech}^{2}\left(\frac{\theta_{1}}{2}\right)$

and the singular one-soliton solution is

$\mathrm{u}(\mathrm{x}, \mathrm{t})=\frac{1}{2} \mathrm{k}_{1}{ }^{2} \operatorname{csch}^{2}\left(\frac{\theta_{1}}{2}\right)$,

where $\theta_{1}$ is defined in (4).

For the phase shift we find that

$$
\mathrm{a}_{12}=\frac{\left(\mathrm{k}_{1-}-\mathrm{k}_{2}\right)^{2}}{\left(\mathrm{k}_{1+} \mathrm{k}_{2}\right)^{2}}
$$

and for other phase shifts we write

$$
a_{i j}=\frac{\left(k_{i}-k_{j}\right)^{2}}{\left(k_{i+} k_{j}\right)^{2}}
$$

As a result, we can eas1y obtain explicit forms for the $\mathrm{N}$-soliton solutions for the Lax Eq.(31)

\section{Conclusion}

In this paper, with the aid of symbolic computation, we have investigated a class of fifthorder evo ution equations. By the Cole-Hopf transformation method combined with Hirota's bilinear method, N-soliton solution and singular N-soliton solution for Eq. (1) have been obtained. Fur-thermore, based on the one-soliton solution in expression (10), we have carried out the parametric analysis in order to investigate the effects of the parameters $\alpha, \beta, \gamma$ on the soliton amplitude, width and velocity.

\section{Acknowledgements}

We would like to thank ZU (Zarqa University) for its support and grant in making this work possible. 


\section{References}

[1] C. D. Scott and R. E. Smalley, J. Nanosci. Nanotechnol, vol. 3, no. 75, (2003).

[2] F. Awawdeh, "New exact solitary wave solutions of the zakharov-kuznetsov equation in the electronpositron-ion plasmas", Appl. Math. Comput., vol. 218, (2012), pp. 7139-7143.

[3] S. Al-Shara, F. Awawdeh and H. M. Jaradat, "Applications of a simplified bilinear method to ion-acoustic solitary waves in plasma", Eur.Phys. J. D., vol. 66, (2012), pp. 1-8.

[4] J. Hietarinta, "A search for bilinear equations passing hirota's three-soliton condition. ii. mkdv-type bilinear equations", Math. Phys., vol. 28, (1987), pp. 2094-2101.

[5] Hirota, "Direct methods in soliton theory", R. K. Bullough and P. J. Caudrey, editors, Solitons, Topics in Current Physics, Springer Berlin Heidelberg, vol. 17, (1980), pp. 157-176.

[6] R. Hirota, "Soliton solutions to the bkp equations. i. the pfaffian technique", J. Phys. Soc. Jpn., 58:22852296, (1989).

[7] F. Awawdeh, M. Alquran, H. M. Jaradat and S. Al-Shara, "Variable coefficient equations of the kadomtsevpetviashvili hierarchy: multiple soliton solutions and singular multiple soliton solutions", Physica Scripta, 85:035001, (2012).

[8] M. Ito, "An extension of nonlinear evolution equations of the k-dv (mk-dv) type to higherorde s", J. Phys. Soc. Japan., vol. 49, (1980), pp. 771-778.

[9] D. Kaup, On the inverse scattering problem for the cubic eigenvalue problems of the clas $3 x+6 q x+6 r=$. Stud. Appl. Math., vol. 62, (1980), pp. 189-216.

[10] E. Lax, "Integrals of nonlinear equations of evolution and solitary waves",Comm. Pure Appl. Math., vol. 21, (1968), pp. 467-490.

[11] J. D. Gibbon, P. J. Caudrey and R. K. Dodd, “A new hierarchy of korteweg-de yries equations", Proc. Roy. Soc. Lond. Ser A, vol. 351, (1976), pp. 407-422.

[12] A. Ramani and R. Hirota, "The miura transformation of kaup's equation and of mihailov's equation", Phys. Lett. A, vol. 76, (1980), pp. 95-96.

[13] J. Satsuma and R. Hirota, "Soliton solutions of a Coupled korteweg-devries equation", Phys. Lett. A, vol. 85, (1981), pp. 407-408.

[14] J. D. Gibbon and R. K. Dodd, "The prolongation structûre of a higher order korteweg-de vries equation", Proc. Roy. Soc. Lond. Ser A, vol. 358, (1977), pp. 287-296.

[15] K. Sawada and T. Kotera, "A method of finding n-soliton solutions of the kdv and kdv-like equation", Progr. Theoret. Phys., vol. 51, (1974), pp. 1355-1367.

[16] W. Zhuang and W. Hereman, "A macsyma program for the hirota method", 13th World Congress on Computation and Applied Mathematics, vol, 2, (1991), pp. 842-863.

[17] W. Zhuang and W. Hereman symbolic computation of solitons with macsyma", Comput. Appl. Math. II: Differen. Equat., vol. 2, (1992), pp. 287-296.

[18] A. M. Wazwaz, "Multiple-soliton solutions for the boussinesq equation", Appl. Math. Comput., vol. 192, (2007), pp. 479-486.

[19] A. M. Wazwaz, Completely integrable coupled kdv and coupled kp systems", Commun. Nonlinear. Sci. Numer. Simulat.) vol. 15, (2010), pp. 2828-2835.

[20] A. M. Wazwaz, "Multiple soliton solutions for the (2+1)-dimensional asymmetric nizhnik novikov veselov equation", Nonlinear Analysis, vol. 72, (2010), pp. 1314-1318.

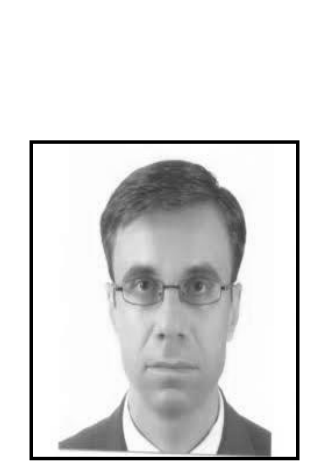

\section{Author}

Dr.Rafat Alshorman, is an assistant professor in the department of computer science at Zarqa University/Jordan. Dr.Alshorman received his $\mathrm{PhD}$ from Loughborough university/Uk, 2009. Dr.Alshorman research interests are: 1. Formal methods 2. Temporal logics 3. Concurrent Databases 4. Serializability of Transaction 5. Numerical analysis. 